\title{
Monitored recurrent glomerular disease after kidney transplantation in adult patient in Mongolia
} \author{
Nanjid Shuhertsend ${ }^{1}$ \\ ${ }^{1}$ Organ Transplantation Center, First Central Hospital of Mongolia, Ulaanbaatar, Mongolia \\ ${ }^{2}$ Kidney Transplantation Team, First Central Hospital of Mongolia, Ulaanbaatar, Mongolia
}

Lkhaakhuu Od-Erdene ${ }^{1}$, Davaadorj Bayn-Undur ${ }^{2}$, Adiya Saruultuvshin² ${ }^{2}$ Tseren Khishgee ${ }^{1}$, Jamba Ariunbold ${ }^{2}$,

Background: Mean cause of end-stage renal disease in Mongolia is chronic glomerulonephritis (CGN). Statistic data of 2019 Health Developing Center in Mongolia showed $65 \%$ of dialysis patients, $69 \%$ of kidney transplantation (KT) patients diagnosed CGN. The reported rate of recurrence of C3 glomerulonephritis (C3GN) after transplantation is over $50 \%$. Aim of our study is to study RGN after KT in adult patient and make recommendation to prevent recurrent glomerulonephritis (RGN) in Mongolia.

Methods: This study included 69\% $(n=239)$ of adult patients who received in Mongolia and in abroad KT with CGN diagnosis with and without native and graft kidney biopsy, and excluded child and retransplanted patients. We monitored the clinical examination and risk factors of the patients last 5 years (2016-2021): age, sex, body mass index (BMI), body pressure, duration time in dialysis, hematuria, proteinuria, lipid, dysproteinemia, glomerular filtration rate, donor type, human leukocyte antigen mismatch, panel reactive antibody, pre- and post-KT kidney biopsy. Study was retrospective review on Microsoft Excel and eHealth program.

Results: Mean age was $36.2 \pm 9.9$ years (18-63), $78 \%$ are males; mean BMI was $26 \pm 8.5 \mathrm{~kg} / \mathrm{m}^{2}, 56 \%$ are hypertensive; in immunological risk factor, $51 \%$ are low risk, $26 \%$ are middle risk. Living donor kidney transplant are $79 \%$ and deceased donor kidney transplant are $21 \% ; 89 \%$ are hematuria, $84 \%$ proteinuria, $59 \%$ hyperlipidemia; mean estimated glomerullar filtration rate was $2.6 \pm 1.8 \mathrm{~mL} / \mathrm{min} / 1.73 \mathrm{~m}$; graft loss from RGN was 8; patient loss was 2. Native kidney biopsy was done only on eight patients and showed five cases of IgA, two cases of MPGN, and one case of focal and segmental glomerulosclerosis. From total 38 graft kidney biopsy, RGN was diagnosed eight cases of IgA and two cases of membranoproliferative glomerulonephritis.

Conclusions: As a result, clinical expertise, early diagnosis, and intervention will help identify recurrent disease and facilitate prompt treatment, thus minimizing graft loss, resulting in improved outcomes. In this review, we highlight the clinic pathological characteristics of certain glomerular diseases that recur in the renal allograft and differentiate from another cause of GN such as virus infection. To decrease the risk factors of RGN in Mongolia, a change of lifestyle is recommended.

Corresponding author: Lkhaakhuu Od-Erdene

E-mail: Ikhaakhuuoderdene@gmail.com

(C) The Korean Society for Transplantation

This is an Open Access article distributed under the terms of the Creative Commons Attribution Non-Commercial License (http://creativecommons.org/licenses/by-nc/4.0/) which permits unrestricted non-commercial use, distribution, and reproduction in any medium, provided the original work is properly cited. 